\title{
Detection of Chlamydia trachomatis in rectal specimens in women and its association with anal intercourse: a systematic review and meta-analysis
}

\author{
Nastassya L Chandra, ${ }^{1,2}$ Claire Broad, ${ }^{3}$ Kate Folkard, ${ }^{1}$ Katy Town, ${ }^{1}$ \\ Emma M Harding-Esch, ${ }^{1,3,4}$ Sarah C Woodhall, ${ }^{1}$ John M Saunders, ${ }^{1}$ S Tariq Sadiq, 1,3,5 \\ J Kevin Dunbar ${ }^{1}$
}

\begin{abstract}
- Additional material is published online only. To view please visit the journal online (http://dx.doi.org/10.1136/ sextrans-2017-053161)

${ }^{1}$ HIV and STI Department, Public Health England, London, UK ${ }^{2}$ Field Epidemiology Service, Public Health England, London, UK

${ }^{3}$ Applied Diagnostic Research and Evaluation Unit, Institute for Infection and Immunity, St George's University of London, London, UK

${ }^{4}$ Research Department of Infection and Population Health, St George's University of London, London, UK

${ }^{5}$ St George's University Hospitals NHS Foundation Trust, London, UK
\end{abstract}

Correspondence to Nastassya L Chandra; nastassya. chandra@phe.gov.uk

Received 20 February 2017 Revised 7 December 2017 Accepted 15 January 2018 Published Online First 3 February 2018

Check for updates

To cite: Chandra NL, Broad C, Folkard K, et al. Sex Transm Infect 2018:94:320-326.

\begin{abstract}
Objectives Chlamydia trachomatis is the most commonly diagnosed bacterial STI. Lack of prevalence and risk factor data for rectal chlamydia in women has testing and treatment implications, as azithromycin (a first-line urogenital chlamydia treatment) may be less effective for rectal chlamydia. We conducted a systematic review of studies on women in high-income countries to estimate rectal chlamydia prevalence, concurrency with urogenital chlamydia and associations with reported anal intercourse (AI).
\end{abstract}

Design Systematic review and four meta-analyses conducted using random-effects modelling.

Data sources Medline, Embase, Cumulative Index to Nursing and Allied Health Literature, PsycINFO and the Cochrane Database were searched for articles published between January 1997 and October 2017.

Eligibility criteria Studies reporting rectal chlamydia positivity in heterosexual women aged $\geq 15$ years old in high-income countries were included. Studies must have used nucleic acid amplification tests and reported both the total number of women tested for rectal chlamydia and the number of rectal chlamydia infections detected. Conference abstracts, case reports and studies with self-reported diagnoses were excluded. Data extracted included setting, rectal and urogenital chlamydia testing results, Al history, and demographics.

Results Fourteen eligible studies were identified, all among diverse populations attending sexual health services. Among routine clinic-attending women, summary rectal chlamydia positivity was $6.0 \%(95 \% \mathrm{Cl}$ $3.2 \%$ to $8.9 \%$ ); summary concurrent rectal chlamydia infection was $68.1 \%$ in those who tested positive for urogenital chlamydia ( $95 \% \mathrm{Cl} 56.6 \%$ to $79.6 \%$ ); and of those who tested negative for urogenital chlamydia, $2.2 \%(95 \% \mathrm{Cl} 0 \%$ to $5.2 \%)$ were positive for rectal chlamydia. Reported Al was not associated with rectal chlamydia (summary risk ratio $0.90 ; 95 \% \mathrm{Cl} 0.75$ to 1.10).

Conclusions High levels of rectal chlamydia infection have been shown in women with urogenital chlamydia infection. The absence of association between reported Al and rectal chlamydia suggests Al is not an adequate indicator for rectal testing. Further work is needed to determine policy and practice for routine rectal testing in women.

\section{INTRODUCTION}

Chlamydia trachomatis (CT) is the most commonly diagnosed bacterial STI in high-income and middle-income countries, primarily infecting urogenital and rectal mucosa. ${ }^{12}$ Failure to identify and treat urogenital CT can result in serious sequelae such as pelvic inflammatory disease, tubal scarring, ectopic pregnancy and infertility. ${ }^{13}$ Rectal CT infection is largely asymptomatic, and is associated with increased risk of HIV transmission and acquisition. ${ }^{45}$ High concurrency of rectal and urogenital CT in men and women has been observed in some studies, suggesting significant numbers of rectal CT cases may be missed by genital testing alone. ${ }^{6-9}$

The clinical significance of rectal CT infection in women is unclear, as the risks of long-term sequelae following a rectal infection in women are unknown. ${ }^{9}$ A positive nucleic acid amplification test (NAAT) result may not represent viable organisms emerging from rectal mucosal intracellular CT infection (a true infection), and instead be indicative of contamination of either DNA or CT organisms from the urogenital tract to the rectum. However van Liere et $a l^{10}$ have shown comparable bacterial loads in rectal and in urogenital swabs, supporting the premise that detection of CT in the rectum can indicate a biological infection rather than contamination in women with and without a history of anal intercourse (AI). Henceforth, we assume that a positive test means an established CT infection. Undiagnosed, untreated and undertreated rectal CT infections constitute a potential reservoir of CT, and given the possibility of autoinoculation of CT from the rectum to the urogenital tract ${ }^{1112}$ may impede effective treatment and transmission prevention.

A recent meta-analysis and other work suggest that azithromycin, a first-line treatment for uncomplicated urogenital CT, may only be around $80 \%$ effective when treating rectal CT. ${ }^{2} 1314$ Consequently, the WHO and the BASHH recommend doxycycline as the preferred treatment for rectal CT, with the former specifically recommending doxycycline if AI is reported. ${ }^{2} 15$ In England, 11\% of women aged 16-74 years reported AI in the previous year. There is inconsistent evidence for history of AI as a rectal CT indicator. ${ }^{9}{ }^{16-20}$ However, WHO guidelines currently recommend that a history of AI guide the decision to test for rectal CT. ${ }^{15}$

Many countries have policies for opportunistic urogenital CT testing and treatment, such as the National Chlamydia Screening Programme in England, which tests approximately 1.7 million people aged 15-24 and identifies around 140000 
infections annually. ${ }^{12122}$ Having unidentified reservoirs of rectal CT in women tested only for urogenital CT risks reduced effectiveness and cost-effectiveness of these CT control programmes.

We conducted a systematic review and meta-analyses of studies measuring rectal CT positivity in women in order to estimate rectal CT prevalence, concurrency with urogenital CT and the association between rectal CT and reported history of AI.

\section{METHODS}

\section{Search strategy and selection criteria}

We searched electronic bibliographic databases-Medline, Embase, Cumulative Index to Nursing and Allied Health Literature, PsycINFO and the Cochrane Database-from 1 January 1997 to 2 September 2015 using free-text terms and medical subject headings combining all terms for CT, prevalence, positivity, epidemiology, diagnosis, and rectal, anal or anogenital (online supplementary material 1). Additionally, we hand-searched references of included papers for other relevant papers. Conference abstracts were not included. Using the same method, we undertook an additional Medline search between 1 January 2015 and 17 October 2017 to ensure that all relevant recently published articles were included in the review.

We included cross-sectional studies of general and clinic-attending populations, which could be nested in cohort studies or randomised controlled trials, involving heterosexual women aged $\geq 15$ years who were tested for rectal CT. Studies must have used NAATs and reported both the total number of women tested for rectal CT and the number of rectal diagnoses. Case reports and studies with self-reported diagnoses were excluded. Studies were limited to those conducted in high-income countries (defined by the Organisation for Economic Co-operation and Development), as these countries were most likely to have similar healthcare provision and CT epidemiology.

Abstracts and full texts were independently reviewed by two reviewers for eligibility. Disagreements were resolved with a third reviewer. Where necessary, study authors were contacted for more information.

Data were extracted independently by two reviewers, compared and discrepancies resolved with a third reviewer. Data extracted included information on study design, inclusion criteria, outcome of interest and funding information (online supplementary material 2). Urogenital and rectal infections were defined as a positive NAAT from a site-specific swab which was not part of a pooled sample. We assume that a positive rectal swab for CT in a woman represents an active and established rectal CT infection. Data were extracted from papers that reported any history of AI by rectal CT test result (AI not specifically defined).

Risk of bias was assessed by two independent reviewers using a published tool adapted from Hoy et $a l^{23}$ to determine whether included studies would present a biased estimate of population prevalence. The tool assesses risk of bias using structured questions to appraise internal and external validity of each study. The reviewers collated their evaluations of each paper and agreed on an overall risk for each paper.

\section{Data analysis}

The positivity of rectal infection among participants in each study was calculated, defined as percentage of women with a rectal CT infection among all women in the study tested for rectal CT.
Four meta-analyses were conducted, using random-effects models to calculate the following:

1. summary estimates of rectal CT positivity across all studies stratified by tested population or clinical subgroup: (1) routine clinic attenders (not defined as high-risk as below), $n=3$; (2) women of high risk (sexual contacts of gonorrhoea-positive individuals, had symptoms, victims of sexual assault, had sexual contact with someone diagnosed with CT, or were being followed up for CT and adult film industry performers), $n=4$; (3) women who were tested only because they reported a history of AI, $n=5$; (4) women who were tested for rectal CT because they were positive for urogenital CT, $n=1$; and (5) women who were all HIV-positive, $n=1$

2. summary estimates of proportion of women with rectal CT among women who were positive for urogenital CT in all appropriate studies and among studies reporting routine clinic attenders; these women represent a population who may have an undiagnosed rectal CT infection but have treatment directed towards their urogenital CT infection which may not be the recommended first-line treatment for rectal CT (doxycycline), hereafter we refer to this as 'less effectively treated'

3. summary estimates of proportion of women with rectal CT among those negative for urogenital CT in all appropriate studies and among studies reporting routine clinic attenders, as a measure of potential undiagnosed rectal infection among those tested

4. summary risk ratio (RR) for being rectal CT-positive in women who had reported a history of AI in order to determine the utility of reporting $\mathrm{AI}$ as an indicator for rectal testing.

Where heterogeneity was high (more than 75\%), summary estimates are not reported (with exception of routine clinic-attending women), and only a range and median average of the results are reported.

Additional data were extracted on specimen type and site tested (online supplementary material 3), but were not analysed to determine association with positivity.

All meta-analyses were undertaken using STATA V.13, using the metaprop (analyses 1-3) and metan (analysis 4) commands. We tested for variation in estimated proportions (analyses 1-3) or RR (analysis 4) attributable to heterogeneity using the $I^{2}$ statistic and estimated between-study variance using the $T^{2}$ statistic. A fixed continuity correction of 0.5 was added in cases where a study had a zero result, to ensure all studies could be included, where appropriate, in analyses. ${ }^{24} 25$

\section{RESULTS}

From 681 unique references identified from the database search, 14 studies were included in the meta-analyses (figure 1): 12 cross-sectional studies and 2 observational cohort studies (table 1). Of these 14 studies, 5 only tested women for rectal CT if they had reported a history of $\mathrm{AI}^{826-29} ; 1$ conducted only among HIV-positive women ${ }^{30} ; 1$ conducted only among urogenital CT-positive participants ${ }^{9} ; 1$ conducted among adult film industry performers ${ }^{31}$; 3 studies tested clinic attendees considered to be high risk (defined as women who were sexual contacts of gonorrhoea-positive individuals, had symptoms, were victims of sexual assault, had sexual contact with someone diagnosed with CT or were being followed up) ${ }^{73233}$; and 3 studies included all eligible routine clinic attendees. ${ }^{16} 1734$ All studies identified were of women attending sexual health settings, and no studies conducted among a general population sample were identified. 


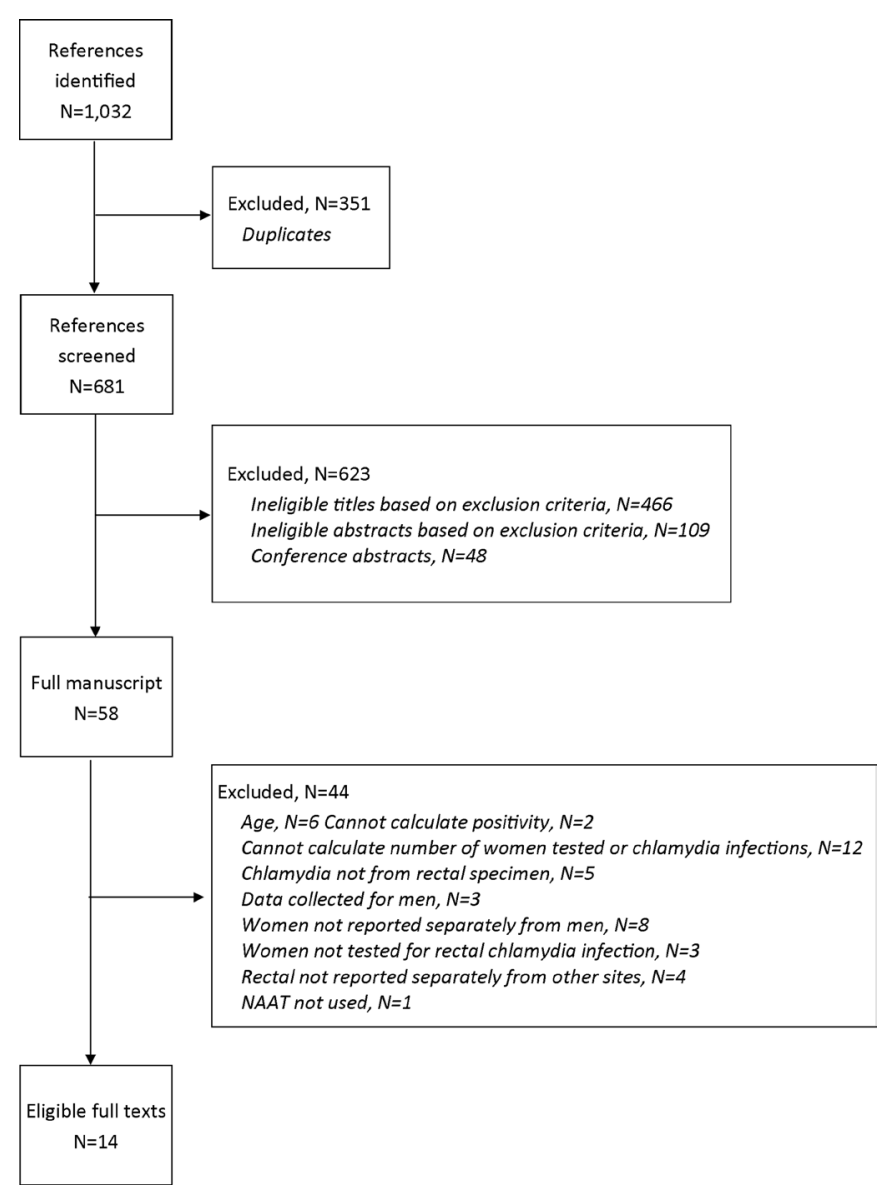

Figure 1 Flow chart depicting the selection of studies for inclusion in the review process. NAAT, nucleic acid amplification test.

Using the adapted risk-of-bias tool, ${ }^{23}$ all studies were considered to have an overall high risk of bias in measuring population prevalence given that they each sampled from specific groups, which is unlikely to represent the general population of sexually active women in their respective countries. Some answers varied by reviewer, but the overall risk was found to be the same (online supplementary material 4).

No study provided an estimated population prevalence of rectal CT. Among all 14 studies, rectal CT positivity ranged from $1.7 \%$ to $77.3 \%$ with a median of $8.9 \%$ (table 1 ). Due to the high heterogeneity between studies $\left(I^{2}=97.2 \%, T^{2}=0.01\right)$, a summary estimate was not calculated for all studies.

For the studies reporting routine clinic-attending women, although high heterogeneity was seen across the three studies, the subgroup summary estimate for rectal CT positivity was $6.0 \%\left(95 \%\right.$ CI $3.2 \%$ to $\left.8.9 \%, I^{2}=84.6 \%, T^{2}=0.01\right)$. For the studies where all women reported a history of AI, the summary estimate for rectal CT positivity was $25.9 \%$ (95\% CI $8.5 \%$ to $43.3 \%, I^{2}=65.2 \%, T^{2}=0.00$ ) (figure 2 ).

Ten studies reported testing for urogenital CT and rectal CT. $^{7-9} 161727$ 31-34 Among these studies where all women were tested for rectal CT and urogenital CT, a concurrent infection was found in 3.6\%-81.5\% (median, 11.8\%) (table 2). Among studies where women were positive for CT regardless of site of infection, concurrent urogenital CT and rectal CT was found in 40.9\%-100\% (median, 78.5\%; calculated using columns from table $2 \mathrm{~A} /(\mathrm{A}+\mathrm{B}+\mathrm{C}))$. In studies in which women were positive for rectal CT, a concurrent urogenital CT infection was found in 62.9\%-100\% (median, 94.3\%; A/(A+C)) (table 2).

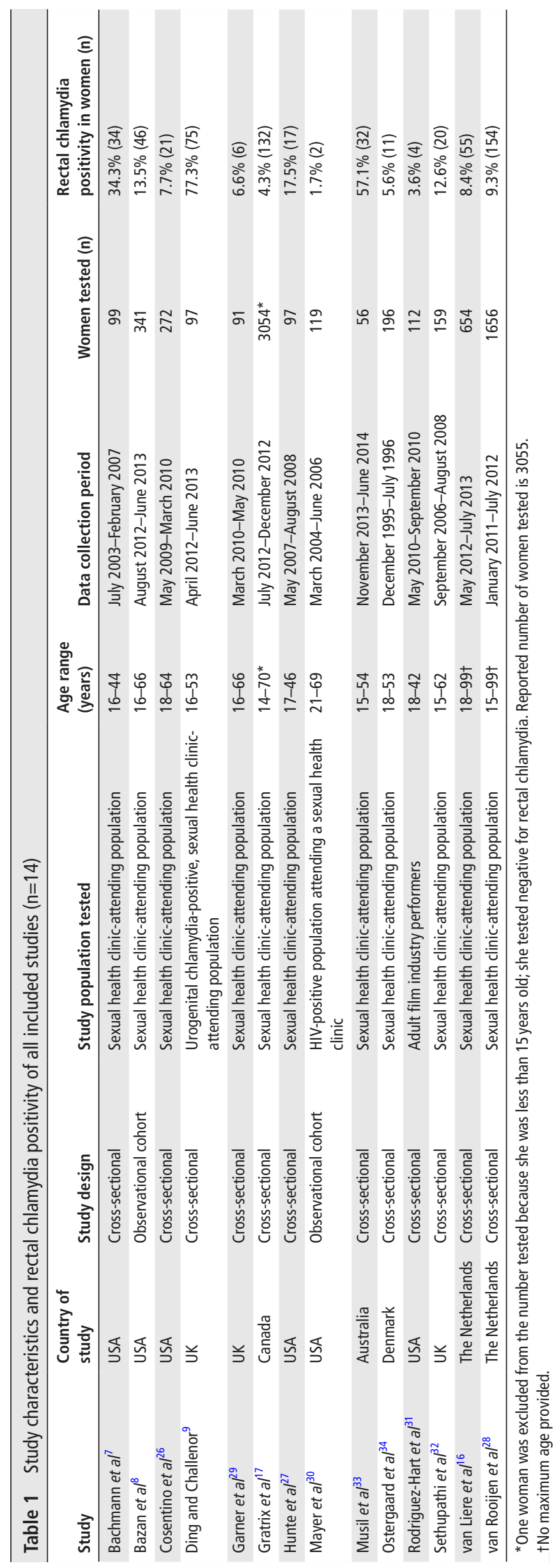


b) Women of high risk* Musil et al., 2016 Rodriguez-Hart et al., 2012 Sethupathi et al., 2010 Bachmann et al., 2010 Subtotal

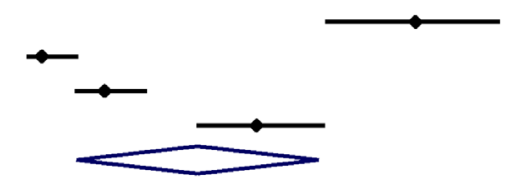

$0.571(0.441,0.692) \quad 23.14$ $0.036(0.014,0.088) \quad 26.27$ $0.126(0.083,0.186) \quad 25.94$ $0.343(0.257,0.441) \quad 24.65$ $0.259(0.085,0.433) \quad 100.00$

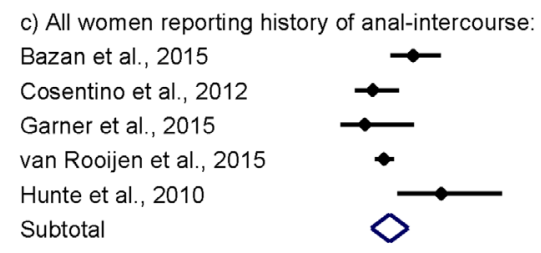

$0.135(0.103,0.175) \quad 21.02$ $0.077(0.051,0.115) \quad 23.15$ $0.066(0.031,0.136) \quad 15.20$ $0.093(0.080,0.108) \quad 31.54$ $0.175(0.112,0.263) \quad 9.09$ Subtotal $0.102(0.075,0.128) \quad 100.00$

d) All positive for urogenital $\mathrm{CT}$ : Ding and Challenor, 2013

$0.773(0.680,0.845) \quad 100.00$

e) All HIV positive women: Mayer et al., 2012 $0.017(0.005,0.059) \quad 100.00$

Figure 2 Individual study and study subgroup summary estimates of rectal chlamydia positivity in women stratified by clinical subgroup/population type $(n=14)$. The diamonds represent the summary proportions and Cls by subgroup. *Women of high risk category includes sexual contacts of gonorrhoea-positive individuals, had symptoms, were victims of sexual assault, had sexual contact with someone diagnosed with CT or were being followed up, as well as adult film industry performers (potentially high risk due the number of sexual events, however safe-sex and sexual health testing practice is unknown). CT, Chlamydia trachomatis.

High heterogeneity was found between the 10 studies $\left(I^{2}=80.9 \%, T^{2}=0.01\right)$. The proportion of women having a rectal CT infection among those positive for urogenital CT ranged between $45.0 \%$ and $100 \%$. This range represents a worstcase scenario of women at risk of being less effectively treated (ie, women with undiagnosed rectal CT who do not receive the recommended first-line treatment for rectal CT) (online supplementary material $5 \mathrm{a}$ ). The summary estimate of rectal CT infection among those positive for urogenital CT across studies reporting only routine clinic attenders was $68.1 \%$ (95\% CI $56.6 \%$ to $\left.79.6 \%, I^{2}=70.9 \%, T^{2}=0.03\right)$.

A summary estimate among those who tested negative for urogenital CT was not provided due to the high heterogeneity $\left(I^{2}=91.0 \%, T^{2}<0.0001\right)$. The proportion of women with a rectal CT infection among women who tested negative for urogenital CT ranged from $0.0 \%$ to $11.5 \%$. This range represents the potential proportion of women with a CT infection who may have been undiagnosed and untreated (online supplementary material $5 \mathrm{~b}$ ). Although a high heterogeneity was found, the summary estimate for studies reporting only routine clinic attenders was 2.2\% (95\% CI $0 \%$ to $5.2 \%$, $I^{2}=97.3 \%, T^{2}=0.001$ ).

Eleven studies had information on history of AI. ${ }^{7-9161726-2932-34}$ The definition of 'history of AI' was inconsistent across the studies or not specified. Among all women who were tested for rectal CT, 2.0\%-30.4\% (median, 9.1\%; F/J) had a positive test and reported AI (table 2). Among all women who had a positive rectal CT infection, between $13.5 \%$ and $100 \%$ (median, $51.6 \% ; \mathrm{F} /(\mathrm{F}+\mathrm{H})$ ) reported AI. However these included five studies that only tested women who reported AI; without these five studies, ${ }^{86-29}$ the range is $13.5 \%-50.0 \%$ (median, $29.1 \%$ ) (table 2).

The calculated summary RR for a history of AI as a risk factor for rectal CT was 0.90 (95\% CI 0.74 to $1.10, I^{2}=5.6 \%$, $\left.T^{2}=0.004\right)$ (online supplementary material 6).

\section{DISCUSSION}

The systematic review and meta-analyses of 14 studies found a summary estimate of rectal CT positivity of $6.0 \%$ among routine clinic-attending women in high-income countries. 


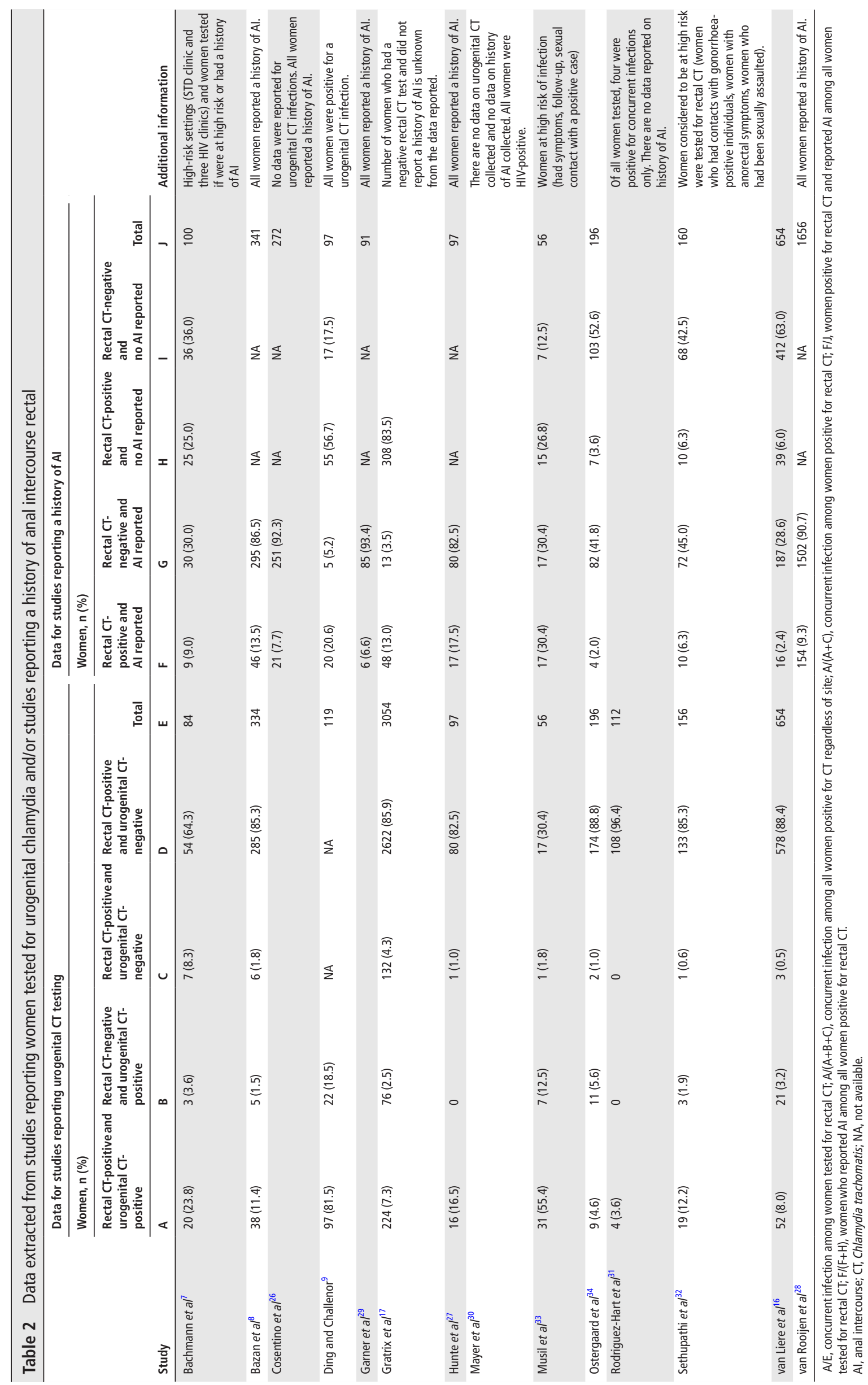


However, because of high heterogeneity and bias due to the populations sampled, this is a likely overestimate of population prevalence of rectal CT in women. We calculated that $68.1 \%$ of routine clinic-attending women infected with urogenital CT also had rectal CT, which is important for treatment implications, as azithromycin is a recommended first-line treatment for urogenital CT but is less effective for rectal CT. Of routine clinic-attending women without urogenital CT, $2.2 \%$ had a rectal CT infection, which would go undetected if they only have urogenital testing. Most interestingly, this analysis did not find a relationship between rectal CT and reported AI, and therefore we believe that currently there is insufficient evidence to guide practice on using reported $\mathrm{AI}$ as an indicator for rectal testing, as recommend by WHO guidelines. ${ }^{15}$

A key strength of our study is that we employed a robust methodology to search for and review papers following an a priori protocol with clear inclusion and exclusion criteria. We searched several databases, assessed papers for risk of bias and undertook double extraction. Our findings are subject to limitations, arising from the nature of included studies. First, estimates cannot be applied to the general population as all 14 studies included women attending sexual health services, limiting the generalisability. Second, sexual health clinic populations varied, thus limiting comparability. Third, there was no consistent definition of history of AI so findings should be interpreted with caution. Similarly, 'high-risk' women were inconsistently defined.

This is the first systematic review of the prevalence of rectal CT in heterosexual women in high-income countries. A non-systematic review of the literature on extragenital infections showed similar findings for rectal CT positivity and also highlighted that extragenital infections are often found in the absence of reported risk behaviours such as AI. ${ }^{6}$ However, the authors did not calculate an estimate for concurrent urogenital CT or RR for AI and rectal CT.

The uncertainty regarding rectal CT infections representing true infections versus contamination could not be taken into account in this review given that included studies did not undertake any testing method to rule out contamination.

Although questions remain about the meaning of a rectal CT diagnosis, our study raises some important issues concerning CT testing and treatment policies. We found that a summary estimate of $68.1 \%$ of routine clinic-attending women with urogenital CT had concurrent rectal CT. While the relative efficacy of azithromycin versus doxycycline for rectal CT in women has not been definitively established, a high proportion of routine clinic-attending women may be subject to less effective treatment, if given azithromycin directed at their urogenital infection in the absence of rectal testing. Assuming our estimate to be true and combining it with a previous estimate of azithromycin effectiveness for rectal CT of $82.9 \%$ (95\% CI $76.0 \%$ to $89.8 \%)^{14}$ suggests that among routine clinic-attending women diagnosed with urogenital CT, only approximately half ( $56 \%$; calculated by $68.1 \%$ multiplied by $82.9 \%)$ would receive adequate treatment if their rectal infection remained undiagnosed and they had received azithromycin. Furthermore in approximately $12 \%$ (calculated by $17.1 \%$ multiplied by $68.1 \%$ ), treatment would have failed. In genitourinary medicine clinics in England 2015, there were 794168 CT tests and 50708 diagnoses reported among women (all ages). ${ }^{35}$ Applying our results to this surveillance data shows the potential scale of less effective treatment (diagnoses multiplied by $56 \%$ ) in women attending sexual health services. If we assume all tests and diagnoses were for urogenital CT only, and that azithromycin is prescribed, in this scenario, approximately 28400 CT infections may have been less effectively treated and approximately 6000 infections would fail to be treated (diagnoses multiplied by $12 \%$ ). However, in practice, some clinicians are likely to further assess the need for rectal CT testing (eg, through history of AI) and manage accordingly so fewer infections are likely to be at risk of less effective treatment.

Furthermore, our finding that an estimate of $2.2 \%$ of routine clinic-attending women without a urogenital CT infection had rectal CT suggests a number of rectal CT infections could be missed even among those actively engaged in testing. Among all studies with available data, only $13.5 \%$ of rectal CT detected were among women reporting AI. Therefore, limiting testing and treating for rectal CT to women reporting a history of AI or rectal symptoms (as recommended by current guidelines) could miss a significant number of rectal infections, which has potential implications for current CT control programmes.

These findings suggest that less effective treatment and missed diagnoses in women may be occurring on a considerable scale. However the clinical significance of missed and untreated rectal CT in women is still uncertain. Furthermore, the potential impact of less effective treatment may not be as great because in practice, some women receive doxycycline as first-line treatment for urogenital CT; currently this proportion is unknown.

From our findings it is clear that current evidence is insufficient to make a robust recommendation regarding routine rectal CT testing in women across settings and the use of history of $\mathrm{AI}$ as a reliable indicator, highlighting the need for further research. While the evidence is limited, rectal testing could be done in women who repeatedly present with a urogenital infection within 3-6 months of treatment. Current BASHH guidance in the UK on the management of CT infection states that those who test positive should be retested after 3 months to identify reinfection. ${ }^{2}$ Positive tests at this stage could be tested for rectal infection or simply treated for rectal infection. High rates of concurrent rectal and urogenital CT are not enough to warrant the use of doxycycline rather than azithromycin for first-line treatment of women with urogenital CT; however, a randomised controlled trial of azithromycin versus doxycycline in women for the treatment of rectal CT would determine whether individuals with a diagnosed urogenital CT infection but undiagnosed rectal CT who are treated with azithromycin are being treated suboptimally. Data on current prescribing (azithromycin or doxycycline for urogenital CT) and extragenital testing practices in specialist and non-specialist settings would add understanding to the scale of potential less effective treatment. High-income countries with national testing and treatment policies also require a robust estimate of rectal CT among their target populations. Biological studies on bacterial viability as well as non-urogenital sources

\section{Key messages}

- Currently there are no definitive estimates of the prevalence of rectal chlamydia, of concurrent rectal and urogenital chlamydia infection, or of the relationship between anal intercourse and rectal chlamydia infection.

- This systematic review found high rates of concurrent rectal infection in women with urogenital infection and no evidence that a history of anal intercourse is a reliable indicator for rectal chlamydia infection.

- Using reported anal intercourse as an indicator for rectal testing is likely to lead to missed diagnoses of rectal infections. 
of rectal CT infection such as orally acquired ${ }^{12} 36$ would further allow implications of rectal CT to be better understood. Studies to ascertain feasibility, costs and acceptability of different testing strategies are also needed to understand how rectal testing may best be incorporated into clinical pathways. It would be interesting to determine if other clinical practices such as who obtains the sample, specimen type and the invasiveness of the swab affect the positivity rate of rectal chlamydia. Finally, there is a need for studies to determine the significance of rectal CT in women by understanding the natural history and complications associated with rectal CT.

In conclusion, we have found evidence that a substantial proportion of women attending sexual health clinics are infected with rectal CT and that the infections risk being missed or less effectively treated. Further work is needed to determine the feasibility of and criteria for routine rectal testing in women.

\section{Handling editor Nicola Low \\ Contributors SCW, STS, JMS, EMH-E, KF and JKD had the initial idea and concept of undertaking this systematic review. JMS and SCW developed the idea and wrote the study protocol which laid out the search strategy and study design. NLC undertook the literature search and reviewed the titles. NLC, KT, CB and KF reviewed the abstracts and full-text papers. JMS and JKD evaluated the included papers for risk of bias. NLC, CB and KF extracted and collected the data. NLC designed and undertook the meta-analyses, with contributions from all authors. NLC wrote the first draft of the paper and developed the figures. All authors contributed to the writing of the manuscript and approved the final version.}

Funding Project funded by Public Health England, the UKCRC Translational Infection Research (TIR) Initiative supported by the Medical Research Council, eSTI2 Consortium (grant number G0901608), and the National Institute for Health Research (NIHR) i4i Programme (grant number II-LB-0214-20005). The funding bodies had no role in the design of the study, in the writing of the manuscript and in the decision to submit the manuscript for publication. The views expressed are those of the authors and not necessarily those of the NIHR, the NHS or the Department of Health.

\section{Competing interests None declared.}

Provenance and peer review Not commissioned; externally peer reviewed.

Data sharing statement No additional data available.

(c) Article author(s) (or their employer(s) unless otherwise stated in the text of the article) 2018. All rights reserved. No commercial use is permitted unless otherwise expressly granted.

\section{REFERENCES}

1 Public Health England. Opportunistic chlamydia screening of young adults in England: an evidence summary. London, UK: Public Health England, 2014

2 Nwokolo NC, Dragovic B, Patel S, et al. 2015 UK national guideline for the management of infection with Chlamydia trachomatis. Int J STD AIDS 2016:27:251-67.

3 Price MJ, Ades AE, Soldan K, et al. The natural history of Chlamydia trachomatis infection in women: a multi-parameter evidence synthesis. Health Technol Assess 2016;20:1-250

4 Koedijk FD, van Bergen JE, Dukers-Muijrers NH, et al. The value of testing multiple anatomic sites for gonorrhoea and chlamydia in sexually transmitted infection centres in the Netherlands, 2006-2010. Int J STD AIDS 2012:23:626-31.

5 Cohen MS. Sexually transmitted diseases enhance HIV transmission: no longer a hypothesis. Lancet 1998;351(Suppl 3):S5-S7.

6 Chan PA, Robinette A, Montgomery M, et al. Extragenital infections caused by chlamydia trachomatis and Neisseria gonorrhoeae: a review of the Literature. Infect Dis Obstet Gynecol 2016;2016:1-17.

7 Bachmann LH, Johnson RE, Cheng $\mathrm{H}$, et al. Nucleic acid amplification tests for diagnosis of Neisseria gonorrhoeae and Chlamydia trachomatis rectal infections. J Clin Microbiol 2010;48:1827-32.

8 Bazan JA, Carr Reese P, Esber A, et al. High prevalence of rectal gonorrhea and Chlamydia infection in women attending a sexually transmitted disease clinic. J Womens Health 2015:24:182-9.

9 Ding A, Challenor R. Rectal Chlamydia in heterosexual women: more questions than answers? International Journal of STD and AIDS 2013;24.
10 van Liere G, Dirks J, Hoebe C, et al. Genital and anorectal Chlamydia trachomatis load in women with a concurrent infection 30th IUSTI-Europe Congress. Budapest 2016.

11 Craig AP, Kong FY, Yeruva L, et al. Is it time to switch to doxycycline from azithromycin for treating genital chlamydial infections in women? Modelling the impact of autoinoculation from the gastrointestinal tract to the genital tract. BMC Infect Dis 2015;15:200.

12 Rank RG, Yeruva L. Hidden in plain sight: chlamydial gastrointestinal infection and its relevance to persistence in human genital infection. Infect Immun 2014:82:1362-71.

13 Centers for Disease Control and Prevention. Sexually transmitted diseases treatment guidelines 2015. 2015. http://www.cdc.gov/std/tg2015/default.htm (accessed 31 Oct 2016).

14 Kong FY, Tabrizi SN, Law M, et al. Azithromycin versus doxycycline for the treatment of genital chlamydia infection: a meta-analysis of randomized controlled trials. Clin Infect Dis 2014;59:193-205.

15 World Health Organization. WHO guidelines for the treatment of Chlamydia trachomatis, 2016.

16 van Liere GA, Hoebe CJ, Wolffs PF, et al. High co-occurrence of anorectal chlamydia with urogenital chlamydia in women visiting an STI clinic revealed by routine universal testing in an observational study; a recommendation towards a better anorectal chlamydia control in women. BMC Infect Dis 2014;14:274.

17 Gratrix J, Singh AE, Bergman J, et al. Evidence for increased Chlamydia case finding after the introduction of rectal screening among women attending 2 Canadian sexually transmitted infection clinics. Clin Infect Dis 2015:60:398-404.

18 Javanbakht M, Gorbach P, Stirland A, et al. Prevalence and correlates of rectal Chlamydia and gonorrhea among female clients at sexually transmitted disease clinics. Sex Transm Dis 2012;39:917-22

19 Barry PM, Kent CK, Philip SS, et al. Results of a program to test women for rectal chlamydia and gonorrhea. Obstet Gynecol 2010;115:753-9.

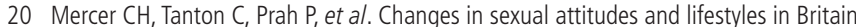
through the life course and over time: findings from the National Surveys of Sexual Attitudes and Lifestyles (Natsal). Lancet 2013:382:1781-94.

21 European Centre for Disease Prevention and Control. Chlamydia control in Europe - a survey of Member States. Stockholm: ECDC 2014;2014

22 U.S. Preventive Services Task Force. Screening for chlamydial infection: U.S. Preventive Services Task Force recommendation statement. Ann Intern Med 2007;147:128-34.

23 Hoy D, Brooks P, Woolf A, et al. Assessing risk of bias in prevalence studies: modification of an existing tool and evidence of interrater agreement. J Clin Epidemio 2012:65:934-9.

24 Friedrich JO, Adhikari NK, Beyene J. Inclusion of zero total event trials in metaanalyses maintains analytic consistency and incorporates all available data. BMC Med Res Methodol 2007:7:5

25 Sweeting MJ, Sutton AJ, Lambert PC. What to add to nothing? Use and avoidance of continuity corrections in meta-analysis of sparse data. Stat Med 2004;23:1351-75.

26 Cosentino LA, Campbell T, Jett A, et al. Use of nucleic acid amplification testing for diagnosis of anorectal sexually transmitted infections. J Clin Microbiol 2012;50:2005-8

27 Hunte T, Alcaide M, Castro J. Rectal infections with chlamydia and gonorrhoea in women attending a multiethnic sexually transmitted diseases urban clinic. Int J STD AIDS 2010;21:819-22.

28 van Rooijen MS, van der Loeff MF, Morré SA, et al. Spontaneous pharyngeal Chlamydia trachomatis RNA clearance. A cross-sectional study followed by a cohort study of untreated STI clinic patients in Amsterdam, The Netherlands. Sex Transm Infect 2015;91:157-64

29 Garner AL, Schembri G, Cullen T, et al. Should we screen heterosexuals for extragenital chlamydial and gonococcal infections? Int J STD AIDS 2015;26:462-6.

30 Mayer KH, Bush T, Henry K, et al. Ongoing sexually transmitted disease acquisition and risk-taking behavior among US HIV-infected patients in primary care: implications for prevention interventions. Sex Transm Dis 2012:39:1-7.

31 Rodriguez-Hart C, Chitale RA, Rigg R, et al. Sexually transmitted infection testing of adult film performers: is disease being missed? Sex Transm Dis 2012;39:987-92.

32 Sethupathi M, Blackwell A, Davies H. Rectal Chlamydia trachomatis infection in women. Is it overlooked? Int J STD AIDS 2010;21:93-5.

33 Musil K, Currie M, Sherley M, et al. Rectal chlamydia infection in women at high risk of chlamydia attending Canberra Sexual Health Centre. Int J STD AIDS 2016:27:526-30.

34 Ostergaard L, Agner T, Krarup E, et al. PCR for detection of Chlamydia trachomatis in endocervical, urethral, rectal, and pharyngeal swab samples obtained from patients attending an STD clinic. Genitourin Med 1997;73:493-7.

35 England PH. Table 4: All STI diagnoses and services by gender and sexual risk. 2011 to 2016;2015.

36 de Vries HJ, Smelov V, Middelburg JG, et al. Delayed microbial cure of lymphogranuloma venereum proctitis with doxycycline treatment. Clin Infect Dis 2009;48:e53-e56. 